\title{
BMJ Open Ageing as a mindset: a study protocol to rejuvenate older adults with a counterclockwise psychological intervention
}

\author{
Francesco Pagnini, ${ }^{01,2}$ Cesare Cavalera, ${ }^{1}$ Eleonora Volpato, ${ }^{1,3}$ \\ Benedetta Comazzi, ${ }^{3}$ Francesco Vailati Riboni, ${ }^{1}$ Chiara Valota, ${ }^{3}$ \\ Katherine Bercovitz, ${ }^{2}$ Enrico Molinari, ${ }^{1,4}$ Paolo Banfi, ${ }^{3}$ Deborah Phillips, ${ }^{2}$ \\ Ellen Langer ${ }^{2}$
}

To cite: Pagnini F, Cavalera C, Volpato $\mathrm{E}$, et al. Ageing as a mindset: a study protocol to rejuvenate older adults with a counterclockwise psychological intervention. BMJ Open 2019;9:e030411. doi:10.1136/ bmjopen-2019-030411

- Prepublication history for this paper is available online. To view these files, please visit the journal online (http://dx.doi org/10.1136/bmjopen-2019030411).

Received 13 March 2019 Revised 16 May 2019 Accepted 12 June 2019
Check for updates

(c) Author(s) (or their employer(s)) 2019. Re-use permitted under CC BY-NC. No commercial re-use. See rights and permissions. Published by BMJ.

${ }^{1}$ Department of Psychology, Università Cattolica del Sacro Cuore, Milano, Italy

${ }^{2}$ Department of Psychology, Harvard University, Cambridge Massachusetts, USA

${ }^{3}$ IRCCS Fondazione Don Carlo Gnocchi, Milan, Italy

${ }^{4}$ Istituto Auxologico Italiano

Istituto di Ricovero e Cura a

Carattere Scientifico, Milano, Italy

Correspondence to

Professor Francesco Pagnini; francesco.pagnini@unicatt.it

\section{ABSTRACT}

Introduction Although ageing is generally perceived as a biologically determined process, the literature increasingly points to the importance of psychological factors in the ageing process, specifically age-related stereotypes or cognitive mindsets. Such stereotypes reflect self-perceptions and others' perceptions about the ageing process and can have a strong influence on health and life satisfaction, specifically through self-fulfilling prophecy mechanisms. This study aimed to investigate whether changes in mindsets can change the ageing process.

Methods and analysis This study replicates in large part the original 1979 'Counterclockwise' experiment by Ellen Langer and will involve a group of older adults (aged 75+) taking part of a 1-week retreat outside of Milan, Italy. Participants will be instructed and helped to relive their younger selves, acting as if they are living in the year 1989. The week-long residential programme is designed to prime this perception by incorporating a completely retrofitted physical environment, as well as providing opportunities to engage in social activities that would have been common in the late 1980s. This 'counterclockwise' intervention will be tested as a randomised control trial comprised of the experimental ('counterclockwise') group, an active control group (same activities, no time manipulation) and a no-treatment group. Ninety participants will be randomly allocated to one of these three conditions. Every participant will be assessed for medical, cognitive, psychological and age appearance at four time points: at the time of recruitment, after the intervention (ie, after a week for the no-treatment group) and again after 6 and 12 months. Ethics and dissemination The study has been approved by the Ethics Committees of the Department of Psychology of Università Cattolica del Sacro Cuore and Don Gnocchi Foundation. Results will be disseminated through peerreviewed journals, scientific meetings and direct presentation to the general population.

Trial registration number NCT03552042; Pre-results.

\section{INTRODUCTION}

The need for a new active ageing paradigm In the European Union, the ratio of people over age 65 to those between ages 15 and 64
Strengths and limitations of this study

- This study explores how changing in mindset could promote changes in the body of older adults.

- It uses a randomised and controlled method, which was never used in the past for this kind of studies.

- Participants and the research personnel interacting with them are blind to the hypothesis.

is projected to increase from $25.4 \%$ to $53.5 \%$ between 2008 and 2060. ${ }^{1}$ Similarly, the US Census Bureau ${ }^{2}$ reported that between the years 2000 and 2010, the rate of growth for the nation's 65-and-older sector surpassed the rate of increase of the entire population. The percentage of the total population that is over the age of 65 is projected to increase from $15 \%$ to $24 \%$ between 2014 and $2060 .^{3}$ In OECD (Organisation for Economic Co-operation and Development) countries, the average life expectancy has exceeded 80 years, which is an increase of 10 years since $1970{ }^{4}$ There is a need to develop innovative ideas that can enable older adults to remain independent and in good health given the increased longevity of older adults. Research has increasingly turned to improving wellbeing in later life, including investigations about how to preserve physical and cognitive functioning, as well as psychological health, although further investigations are warranted. $^{5}$

\section{Ageing: a mere physical process?}

The ageing process is typically explained as a process of inevitable physiological and psychological decline, ${ }^{6}$ as ${ }^{6}[\mathrm{w}]$ hen biological gerontologists and laypersons use the term ageing, they are most often referring to... the progressive deterioration during 
the adult period of life' ${ }^{7}$ This description of inevitable decline does not explain the variability across cultures or in other measures found in older individuals' health, raising a need to develop approaches that include psychological and social factors to better understand the ageing process. There is increasing direct evidence that ageing is governed by a combination of psychological, behavioural and environmental factors within the individual's control. ${ }^{8}$ Over the last 20 years, many studies have demonstrated the importance of age-related stereotypes. ${ }^{9}$ These stereotypes form a key construct to be further explored in this study.

\section{The effects of age-related stereotypes}

Like any label, the term 'elderly' tends to confound a population that can be very different from each otherfor example, the 92-year-old yogi versus the wheelchair user patient with dementia, indiscriminately ascribing a lack of personal control and both physical and mental decline, essentially similar to chronic illness labels. The development of research on ageing stereotypes focuses as well on including negative attributes (eg, being 'forgetful', 'weak', 'rigid'), ${ }^{910}$ as well as positive characteristics (eg, being 'warm'). These terms prime implicit and explicit stereotypes of older adults in both older and younger cohorts.

Stereotypical images of ageing are recalled and used as they were initially learned, and there is evidence to suggest that as a person ages these stereotypes may become a self-perception. ${ }^{11}$ The long-term effects of these self-perceptions were demonstrated by a pair of studies based on data from the Ohio Longitudinal Study of Ageing and Retirement, in which participants aged 50 or older at baseline were followed for over two decades. ${ }^{12}$ Participants with more positive self-perceptions of ageing at baseline had better functional health over the course of the study and lived an average of 7.5 years longer than those with more negative self-perceptions of ageing. These health advantages remained after adjusting for baseline functional health and other relevant variables. ${ }^{13}$ In another study conducted with a large sample of young and middle-aged men, people with negative age stereotypes demonstrated higher chances than average to experience a cardiovascular event over the next four decades. ${ }^{14}$ Similarly, during a 6 -year study conducted in Germany, age stereotypes from older adults were a significantly better predictor of health than vice versa. ${ }^{15}$

\section{The stereotype embodiment theory}

The stereotype embodiment theory ${ }^{6}$ describes the process of how people age as following their underlying stereotypes about older people. For example, individuals from cultures with predominantly strong negative beliefs about older people were more likely to experience memory problems. ${ }^{16}$ Studies have shown that the self-perception of memory decline and the stereotypic beliefs of ageing are reliable predictors of actual memory decline. ${ }^{17}$ Furthermore, perceived memory decline was associated with subsequent global cerebral metabolic decline. ${ }^{18}$ Brain modifications associated with Alzheimer's disease, such as hippocampal volume loss and accumulation of neurofibrillary tangles and amyloid plaques, is correlated with the negative age stereotypes earlier in life ${ }^{19}$ Similarly, positive attitudes towards age protect against dementia, even when there is a genetic disposition. ${ }^{20}$

\section{The 'counterclockwise' experiment and the role of perceived control}

One of the major mechanisms of the stereotype embodiment is the reduction of perceived control, as older adults who feel more in control are able to offset the 'negative' effects of the stereotypes. ${ }^{21}$ In one early study on personal control and ageing in a nursing home, residents who were instructed to think of themselves as more independent and had more responsibility for their daily activities, rather than relying purely on caregiver or nursing staff, lived longer than those who were treated just as nicely but were not provided with activities that would increase their perceived independence. ${ }^{22}$ The study demonstrated a significant improvement in the experimental group over the comparison group on alertness, active participation and a general sense of well-being. ${ }^{22} 23$ Moreover, those afforded more independence were twice as likely to be alive 18 months later. ${ }^{23}$ This and other research took issue with the concept of 'learned helplessness, ${ }^{24}$ introducing 'self-induced dependence', in which helplessness may result from the labels one is given, even when there was prior success on the task in question. ${ }^{25}$ As applied to the elderly, the implication was fairly obvious: if a simple label can induce dependence in a typical work situation (eg, 'assistant' vs 'boss'), then it is similarly apparent that as older adults are put in roles with decreasing expectations for performance, learned helplessness increases, fostering an 'illusion of incompetence'. ${ }^{25}$ As a group, older people stop engaging in activities once performed, they 'bear negative labels', and often end up in positions of dependence, relying on others to do things for them. ${ }^{25}$ This construct of self-induced dependence is one in which a person generalises from the application of an 'inferior' label to one in which (s) he is incompetent, and performance degrades further. Such research supports the idea of a strong mind/body connection, in which the historical Cartesian dualism of mind and body is replaced with a more interactive construct. This concept was first developed and tested in Ellen Langer's 1979 'counterclockwise' study that looked at what effects turning back the clock psychologically would have on an older adult's physiological state. The stated research question was, 'If we put the mind back twenty years, would the body reflect this change?'.

\section{The 1979 'counterclockwise' study}

In the original 1979 'counterclockwise' study, ${ }^{8}{ }^{26}$ the design included eight older men who lived together for 5 days on a retreat as if they were actually living 20 years back in time (ie, in 1959). This 'as if' living construct was critical 
to the design. During the 5 days, the participants were instructed to imagine that it was, indeed, 1959-not to remember what it was like, but to live as if it were 1959. To prime this effect, the researchers provided physical props or elements from the prior era including magazines, viewable television programmes and movies that were popular 20 years earlier. A radio of that era played oldie favourites such as Perry Como and Jack Benny. The researchers facilitated twice-daily discussions about 'current events' (ie, events that happened in 1959) including the need for bomb shelters to protect the country against the Soviet powers and Castro's advance on Havana. Participants were instructed to talk about these events as though they were current events (using the present tense) and not to refer to anything that happened after that date (including personal experiences). Men in the comparison group lived at the same retreat for a week discussing the same topics, but these individuals spent the week reminiscing about these events, using in the past tense in discussion.

This experience resulted in improvements over baseline on several measures, in both groups. ${ }^{27}$ For both groups, hearing, memory and grip strength improved. Photos of participants were rated as appearing significantly younger after the retreat than before. In addition to these, there were further improvements for the experimental group on measures of vision, joint flexibility, manual dexterity, IQ gait, posture and decreased symptoms of arthritis. Despite improvements in both groups, nevertheless, the experimental group outperformed the comparison in all these tasks.

\section{Rationale}

This study's rationale is based on the insight that ageing stereotypes ${ }^{15}$ influence the ageing process itself. Ageing is, at least in part, a reflection of a self-fulfilling prophecy, which reflects one's ageing stereotypes. ${ }^{6} 8$ The current project investigates the effect of a residential week in which participants will experience a 'previous self', which is aimed to contrast with one's prior mindset about ageing self-stereotypes. Once a participant has experienced a different self that is not limited by the self-fulfilling prophecy of the self-perceptions of ageing, he/she may realise that a part of what he/she defined as ageing actually derives from mindsets. Therefore, as further articulated in Langer's framework of the "psychology of possibility', that mindset matters, ${ }^{28}$ the participants should be able to break free from these self-imposed limitations.

\section{Aims}

The project aims to investigate whether changes in mindsets (ie, addressing one's age-related stereotypes) can change several key functional, physical and psychological aspects generally associated with the ageing process. In particular, it is aimed at addressing the negative stereotypes about ageing and promoting a more open view on ageing itself.
The test of the mindset influence occurs by disconnecting the chronological process from a presumed physical declination. The project intends to explore the psychological components of 'getting old' and to attempt to actively reverse these subjective age experiences.

Expected improvements in physical function will be the primary outcome. Medical, cognitive, emotional and appearance variables will be considered secondary outcomes. Stereotypes about age will also be assessed as potential mediators of the effects of the intervention.

\section{METHODS AND ANALYSIS \\ Design}

The proposed study design is a randomised controlled trial testing the efficacy of the Counterclockwise Programme (CCP) for a population of older adults in reducing aspects of physical and psychological decline that are normally associated with ageing. The CCP will be tested in comparison with an active control group that will be exposed to similar stimuli without the 'active substance' of mental time manipulation and with a third, no-treatment control group. Participants from both intervention groups will be assessed before and after programme participation, as well as at 6-month and 12-month follow-up. The same assessment schedule will be followed by the no-treatment group. The anticipated project duration is 2 years.

\section{Participants}

Ninety older adults will be recruited in Milan and nearby areas. Potential participants will be screened according to the following inclusion criteria:

- Seventy-five years or older.

- Absence of cognitive impairment, as assessed with a Mini-Mental State Examination ${ }^{29}$ score $>24 .^{30}$

- Able to commit the time requested to join the retreat (one full week).

- No major disability that involves the use of a wheelchair or technological devices (eg, communication device, invasive or non-invasive ventilation).

- No recent (<9 months) traumatic events (eg, death of a close relative, life-threatening events).

- No traumatic events (eg, death of a close relative, life-threatening events) during the end of the 1980s.

Potential participants will be assessed by a trained psychologist, who will conduct a semistructured interview, either in person or by phone, about their current and previous life experiences (ie, 'In the past 9 months, has something particularly unpleasant happened in your life?'; 'Can you please re-imagine your life during the '80s? What was your job, who you lived with, where...'; 'During the end of the ' 80 s, has something particularly unpleasant happened?'). They will be recruited with advertising in local newspapers, through older adult associations (eg, Università della Terza Età), fliers in general practitioners' offices and social networks (which may reach young people who can inform their older relatives). 


\section{Patient and public involvement}

The public, mainly through older adult associations, had an important role in designing part of the study. In particular, they helped to define the 1989 setting and the activities of the experimental group, as well as the recruitment strategy (where and how to reach the potential sample). Participants who had no role in the study design will be informed about all the obtained results at the end of the study, with a dedicated conference meeting and with written reports.

\section{Randomisation and interventions}

Participants will be randomly assigned to one of the three groups. As couples will be admitted to the study, the two partners will be assigned to the same group. Therefore, a randomised stratification for couple members and 'singles' will be implemented. The randomisation schedule will be generated with a Random Integer Generator, from the website www.random.org (which employs true randomisation from atmospheric noise), with two parallel lists for couples and non-couples.

The three conditions will be:

- Counterclockwise Programme (CCP).

- Active control (AC) group.

- No-treatment control (NTC) group.

Both CCP and AC will take place in the same location. The study will be conducted in a small resort located in the countryside outside Milan (about 1-hour driving), which will be modified according to the research needs. The facility can accommodate participants and research staff, and includes outdoor spaces, private and common rooms, and a big kitchen.

The research staff, who will be responsible for the implementation of the retreat schedules and activities, will be composed of two facilitators and a researcher from the core team. The two facilitators (with an EdD or a similar background), will be blind to the research hypothesis and will only be trained to run one condition. The researcher will have limited interactions with the participants and will supervise the correct implementation of the retreat schedule.

\section{Counterclockwise Programme}

The participants will participate in a 6-day counterclockwise retreat in a retrofitted physical environment circa 1989, which helps the participant to re-experience their younger self.

Groups will be composed of 10-12 participants plus the staff. Participants will live during the week as if they were in 1989, talking about 1989 events as if they were in the present, and avoiding talking about post-1989 events. Everybody will be invited to participate in conversations and discussions about 1989 in the present tense (presente). Furniture, posters, music, television, newspapers and technological instruments will all reflect what was available in 1989. Current technological devices, including mobile phones, will be kept in a locker controlled by the facilitators and phones of the 1980s will be available for urgent rather than daily use. Before arriving, participants will be asked to provide old photos of themselves and to bring their two favourite old books from the ' 80 s and an article of clothing from that era (if not a dress or jacket, it could be could be a hat or costume jewellery).

Participants will be briefed about the nature of the experiment and they will be encouraged to help each other live as if it was 1989. Participants will be told not merely to reminisce about this earlier era, but to 'inhabit' life in that era, making a psychological leap to be the person they were at the end of the 1980s, as demonstrated in the earlier counterclockwise research. We will tell them that we have good reason to believe that if they are successful at this, they also will feel as well as they did in 1989, and that the sooner they can 'let go' of the present and return to that timeframe psychologically, the more fun they will have and the better they will feel. Before going home, at the end of the week, participants will be briefed about the rationale of the experiment and will participate in a group discussion. During the week, participants will engage in social and physical activities as if they were in 1989, with a younger self. The retreat agenda is reported in box 1 .

\section{AC group}

Participants in the AC group will follow the same agenda as the CCP group, without the constant reference to 1989. The intervention will take place in the same location as the CCP, without any specific change. Current technological devices, including mobile phones, will be kept in a locker controlled by the facilitators. External communications will be discouraged, although it will be possible through phones provided by the facilitators. Activities will mirror the ones of CCP, but participants will not live as if they were younger. The agenda will be the same, but no mention to 1989 will be done. All discussions and activities will refer to present days (eg, instead of discussing the open of the Berlin Wall, they can discuss Brexit or Trump presidency).

\section{NTC group}

Non-treated participants will be assessed with the same timeline followed by the other groups. Participants will receive three coupons, for each assessment after the baseline (at T2, T3 and T4) for a one-night break in a location of their choice (among a selection of commercial services).

\section{Outcome measures}

\section{Functional and medical assessment}

Physical function will be assessed with the Short Physical Performance Battery (SPPB), a well-established measure of physical function in older adults. ${ }^{31}$ It assesses strength, gait and balance by examining, respectively, (1) time to rise from a chair and return to the seated position five times; (2) time to walk eight feet; and (3) ability to stand with feet together in the side-by-side, semitandem and tandem positions for $10 \mathrm{~s}$. Possible scores ranged from 


\section{Box 1 Counterclockwise retreat schedule}

\section{Day 1: Monday}

15:00 Arrival

Welcome (cold and warm drinks)

15:30 Social briefing: the rules of the experiment will be explained by a clinical psychologist. Motivational group discussion to reinforce the engagement of participants and presentation of each participant as if he/she were really in the ' 80 s (and parallel drafting of a blackboard containing names and main information of each).

17:00 They will carry their own bags to their room, where they will find a copy of their favourite book from the ' $80 \mathrm{~s}$

19:15 They will enjoy a dinner the facilitators will have prepared for them. During dinner, facilitators start to engage participants with a discussion (one of several) to prime them to re-engage in 1989 (eg, a game 'what is going on in the world' in which they name key politicians, world events, celebrities, inventions from 1989 and surrounding years). The discussion will be conducted using present tense (present).

20:00 They watch 1989 TV news

$20: 30$ During the evening, they will see a movie from late ' 80 s. They will choose among a selection of movies.

\section{Day 2: Tuesday}

8:30 Wake-up with 1989 radio news

9:00 Breakfast made for them and reading a 1989 newspaper

10:00 Walk in the garden and TV programme that was typically broadcasted in Italy in 1989 before lunch (eg, 'II Pranzo è servito' and 'Forum') 11:30 They prepare lunch with the ingredients we will provide, based on participants' recalled memories

12:15 Lunch

15:00 Cineforum: they watch and discuss, all together, a 1989 movie (from a selection of VHS we will provide) or a TV show that engages them

17:30 Participants prepare dinner and watch TV programmes (eg, 'La Ruota della Fortuna')

19:15 Dinner

20:00 They watch 1989 TV news

20:30 Evening - write an essay describing a (1989) recent terrific event that they are describing to a friend/family member as if it was in the present

21:00 Debriefing about the writing experience

\section{Day 3: Wednesday}

\section{8:30 Wake-up with 1989 radio news}

9:00 Breakfast made for them and reading a 1989 newspaper

10:00 Decide what to cook for dinner and for the next meals. Participants will be inspired by a list of items with 1989 prices in Lire (£)—facilitators will buy ingredients (removing expiration dates and not buying articles that were unavailable or uncommon in Italy in 1989-e.g., quinoa) 11:30 Participants will prepare lunch

\section{2:15 Lunch}

15:00 Teach each other something about what they felt expert at (workshops about their experiences-for example, use of the typewriter, sewing machine and so on)

18:00 Participants will prepare dinner and watch TV programmes

19:15 They will have dinner and watch 1989 TV news

20:00 Trivial pursuit game questions from the past (they also create questions)

\section{Day 4: Thursday}

8:30 Wake-up with 1989 radio news

9:00 Breakfast made for them and reading a 1989 newspaper

Continued

\section{Box 1 Continued}

10:00 Discussion about their favourite book from the past (or they will complete the Trivial pursuit game if not finished)

11:30 Lunch prepared by participants

12:15 Lunch

15:00 Reading and discussion of 1989 sports events (eg, F.C. Inter won Serie A, A.C. Milan won Champion's League), of last year's sport events (eg, 1988 Olympics in Seoul), or reminiscence of sports activities conducted in the ' 80 s. The other option is a discussion of 1989 political events (eg, Fall of the Berlin Wall).

18:00 Participants prepare dinner and watch TV programmes

19:15 Dinner

20:00 They watch 1989 TV news

20:30 Old movie or playing a board game (eg, Monopoly, Risk, Memory Cards, Pictionary) or they watch a movie

\section{Day 5: Friday}

8:30 Wake-up with 1989 radio news

9:00 Breakfast made for them and reading a 1989 newspaper

10:00 Treasure hunt, involving crosswords and puzzles, all referred to 1989 (four teams of three participants with four different routes)

11:30 Participants prepare lunch

12:15 Lunch

15:00 Relax and preparation of the stage for a gala dinner (cooked by participants, facilitators as waiters)

20:30 Gala dinner (cooked by participants, facilitators as waiters)

\section{Day 6: Saturday}

8:30 Wake-up with 1989 radio news

9:00 Breakfast made for them and reading a 1989 newspaper

10:15 Final briefing and group discussion about the take-home message 12:30 Return to Milan and quick lunch at Don Carlo Gnocchi Foundation hospital

14:30 Postintervention assessments

0 to 12 , with a higher score indicating better physical performance. Older individuals who receive lower scores on this measure has increased risk of disability, nursing-home placement and mortality. ${ }^{32}$

We will assess cardiac parameters, namely heart rate (HR) and blood pressure (BP). HR will be assessed with a Nonin 2500C-UNIV Universal Charger for 2500 Series with a mercury palm-style tat us, while BP will be measured with three $45 \mathrm{~s}$ readings on the participant's left harm. Systolic and diastolic BP will be recorded and averaged across the three measurements $(\mathrm{mm} \mathrm{Hg})$.

Respiratory outcomes include saturation $\left(\mathrm{SpO}_{2}\right)$ and respiratory rate $(\mathrm{RR})$. Oxygen saturation is a term referring to the fraction of oxygen-saturated haemoglobin relative to total haemoglobin in the blood, and it will be measured with a Nonin 2500C-UNIV Universal Charger for 2500 Series. RR is the number of breaths per minute, which will be collected to determine whether the respirations are normal, abnormally fast, abnormally slow or nonexistent.

The Digit Triplet Test will be used to assess hearing. It is a speech-in-noise test available online and through tablet apps in different languages for reliable large-scale hearing screening, and which correlates highly $(\mathrm{r}=0.77)$ 
with audiometric thresholds. ${ }^{33}$ The signal to noise ratio, reported in decibels, for the $50 \%$ correct speech recognition threshold will be estimated for each ear.

Blood samples will be collected at the baseline and at the two follow-up assessments, and the following outcomes will be analysed:

- Liver function (alanine aminotransferase, aspartate aminotransferase, gamma-glutamyl transferase and bilirubin).

- Kidney function (urea, creatine and dissolved salts).

- Cardiac function (creatine phosphokinase and myoglobin).

- Lipid profile (cholesterol and triglyceride tests).

- Thyroid function (thyroid-stimulating hormone).

- Other tests (cortisol, albumin, uric acid).

\section{Cognitive assessment}

Memory will be assessed with the Babcock Story Recall Test. $^{34}$ In this verbal memory test, a brief story is presented, and immediate recall is obtained. The story is then presented a second time, and after administration of intervening tasks, delayed recall is obtained. To learning effects between the first two assessments, two parallel versions will be used. ${ }^{35}$ Scores for several normative samples have been reported and it is considered a valid method for assessing verbal memory. ${ }^{36}$ Good interrater reliability has previously been reported..$^{34}$

Attentive function will be evaluated with the Dual Task Performance, a pencil-and-paper test of divided attention, which consists of two components, a digit span task and a visuospatial tracking task. Each task is performed both separately and concurrently. ${ }^{37}$

\section{Psychological and well-being assessments}

The Psychological General Well-being Index is a questionnaire that assesses well-being. It is composed of 22 items representing six dimensions: anxiety, depressed mood, positive well-being, self-control, general health and vitality. ${ }^{38}$ It is a widely used tool to assess quality of life, and it has excellent psychometric validity. ${ }^{39}$

Anxiety will be evaluated with the Geriatric Anxiety Inventory, a 20-item measure of anxiety symptoms, specifically developed for older adults. ${ }^{40}$ It has been shown to have adequate internal consistency, test-retest reliability and concurrent validity. ${ }^{40}$

We will assess depression with the Geriatric Depression Scale, a 30-item self-report measure of depressive symptoms, developed for older adults. ${ }^{41}$ The validity and reliability of this tool have been supported through both clinical practice and research. ${ }^{41} 42$

Another assessed variable, considered a mediator, will be ageing stereotypes. These will be evaluated with the open-ended Image-of-Ageing question: 'What are the first five words or phrases that come to mind when you think of an older person?'. ${ }^{16}$ Three independent raters will score responses on a Likert scale ranging from 1 (very negative) to 5 (very positive).

\section{Appearance}

Participants will be photographed at each assessment to evaluate age appearance. The photos will have a neutral (ie, white) background and will have the same instructions: 'please, smile to the camera'. University students will blindly guess the age of every person in each picture. Pictures will be showed online with a random allocation through the Qualtrics suite to at least 200 undergraduate students. The final score for each picture will be the average age guessed by students.

\section{Data analysis}

As the data from the original counterclockwise study have been lost, and published data do not allow us to run a power analysis, we considered the results obtained by Levy and colleagues. ${ }^{43}$ Guided by that study, we expect a significant difference on the primary outcome (physical function, assessed with the SPPB) between the two treated groups after the retreat, with an effect size of $d=0.85$. That means that a sample size of 30 participants in each group will lead to a statistical power of $89 \%(\alpha=0.05$, two-tailed), while groups composed of 23 participants will have a statistical power of $80 \%$.

To preserve statistical power, missing data at follow-up will be handled with the most appropriate imputation methods. ${ }^{44}$ Descriptive analysis will be reported for every variable. Group differences and time changes will be explored with mixed models, accounting for the possible mediating effect of ageing stereotypes on the relationship between treatment and outcomes ${ }^{45}$ Post-hoc analyses will be conducted to compare single groups. Data will be analysed with SPSS version 25 and R software.

\section{Stepwise procedures}

Participants from the three groups will be assessed four times (see table 1): at recruitment (T1, baseline); after completing the intervention (for the two intervention groups), or 1 week after recruitment for the NTC group (T2, post-treatment); a 6-month follow-up (T3); a 12-month follow-up (T4). Blood tests will not be performed at T2, given the short timeframe since T1. A synoptic table of assessments is reported below.

Demographic data will be collected on all participants: age, gender, education, work activity, religious affiliation and clinical history. Changes in the physical function (T2-T1) will be the primary research outcome. Other medical, cognitive, emotional and appearance assessment will be considered as secondary outcome measures. Stereotypes about ageing will also be assessed as potential mediators of the intervention effects.

\section{Anticipated results and potential impact}

Addressing negative stereotypes about ageing is a highly compelling issue, and one that is directly related to older adults' well-being, their social relationships and their interactions with the environment. This proposal is aimed to address these mindsets and cultural expectations to promote a more open view on ageing. By testing 
Table 1 Assessment strategy

\begin{tabular}{|c|c|c|c|c|c|c|}
\hline Assessment domain & Outcome & Instrument & T1 & T2 & T3 & T4 \\
\hline \multirow[t]{8}{*}{ Functional and medical } & Physical function & Short Physical Performance Battery & $x$ & $x$ & $x$ & $x$ \\
\hline & Cardiac parameters & Heart rate and blood pressure & $x$ & $x$ & $x$ & $x$ \\
\hline & Hearing & Digit Triplet Test & $x$ & $x$ & $x$ & $x$ \\
\hline & Liver function & Blood test & $x$ & & $x$ & $x$ \\
\hline & Cardiac function & Blood test & $\mathrm{x}$ & & $x$ & $\mathrm{x}$ \\
\hline & Lipid profile & Blood test & $x$ & & $x$ & $x$ \\
\hline & Thyroid function & Blood test & $x$ & & $x$ & $x$ \\
\hline & Other blood tests & Blood test & $x$ & & $x$ & $x$ \\
\hline Cognitive & Memory & Babcock Story Recall Test & $x$ & $x$ & $x$ & $x$ \\
\hline \multirow{3}{*}{ Psychological and well-being } & Anxiety & Geriatric Anxiety Inventory & $x$ & $x$ & $x$ & $x$ \\
\hline & Depression & Geriatric Depression Scale & $x$ & $x$ & $x$ & $x$ \\
\hline & Ageing stereotypes & Image-of-Ageing Question & $x$ & $x$ & $x$ & $x$ \\
\hline Appearance & Age appearance & Pictures & $x$ & $\mathrm{x}$ & $x$ & $x$ \\
\hline
\end{tabular}

the influence of mindset on ageing by disconnecting the chronological process from a presumed physical declination, the project intends to explore the psychological components of 'getting old,' and to try to reverse them purposefully. We expect that the results will constitute a significant contribution to the knowledge of the ageing process, in a field (psychological ageing mechanisms) that is sometimes underestimated by gerontologists. Furthermore, the study is designed to provide tools and strategies that can be directly used to address the psychological components of ageing, promoting both physical and psychological well-being.

As mentioned, therefore, the social relevance of the scientific debate about ageing is high. A common thread in this area deals with how we can promote 'active ageing'. ${ }^{46}$ According to the WHO, this concept refers to the process of optimising opportunities for health, participation and security to enhance quality of life as people age. It applies to both individuals and population groups. ${ }^{47}$ According to this view, elders' well-being relies on them staying active in later life; from this perspective, staying active is key to successfully ageing. ${ }^{48}$ Active ageing allows people to realise their potential for physical, social and mental well-being throughout the life course and to participate in society, while providing them with adequate protection, security and care as needed. ${ }^{49}$

In this social scenario, a change in perspective about ageing, from the narrow biomedical perspective to a more open and mindful idea, ${ }^{50}$ based on the psychology of possibility, can actually improve older adults' wellbeing. Challenging the dominant biomedical view, and properly communicating it through various channels, will lead to change mindsets and stereotypes related to the ageing condition. That, in turn, will lead to an improvement in perceived control and empowerment, which by itself is a booster of quality of life. In fact, the awareness that ageing is (at least partially) a mental event that can be reversed can promote a totally different perception of this process. In short, the project has the potential of a remarkable effect on the ageing society, possibly leading to a change in perception of older adults also by younger people.

Together with the extended social impact, a deliverable of the project is the availability of the protocol and the possibility to easily reproduce it. The intervention can be replicated and adapted to different settings, in both research and clinical/social contexts. For example, institutions for older adults and rehabilitation centres, as well as older adult organisations and associations, can set up counterclockwise experiences, following the guidelines that the project will provide. Given that the costs to deliver this treatment can be limited, if any, in the case of an organisation that already organises older adult retreats, the potential number of involved people can be particularly relevant.

Finally, if the hypothesis is confirmed by study results, the scientific impact on both fields of psychology and medicine will further promote the idea that mind and body are deeply interrelated. Despite the widely cited invitation for a biopsychosocial approach, ${ }^{51}$ too often mental/social and physical domains are compartmentalised. High-quality studies on this integration process can challenge the dominant biomedical paradigm, unravelling new scientific knowledge and treatment opportunities. 


\section{Ethics and dissemination}

The study was approved by the Ethics Committees of Università Cattolica del Sacro Cuore (cod. 1-18) and Don Gnocchi Foundation (ref. 16-04-2018), and it has been registered in the repository Clinicaltrials.gov (Identifier: NCT03552042). Informed consent will be collected from all participants. Only the principal investigator and his team will have access to the data. The dissemination process will consider both scientific, policy makers and general population audiences. Scientific dissemination will include publications in peer-reviewed journals, and results will be presented to scientific meetings, in the area of psychology and gerontology.

Contributors FP is the study principal investigator and contributed to develop the study design and to write the protocol. CC, EV, FVR, BC, CV, KB, EM, PB, DP and $\mathrm{EL}$ contributed to develop the study design and to write the protocol. All authors approved the final manuscript.

Funding This project has been supported by a grant from Fondazione Cariplo, Italian private foundation (call 'Ricerca sociale - 2017', rif. 2017-0954).

Competing interests None declared.

Patient consent for publication Not required.

Provenance and peer review Not commissioned; externally peer reviewed.

Open access This is an open access article distributed in accordance with the Creative Commons Attribution Non Commercial (CC BY-NC 4.0) license, which permits others to distribute, remix, adapt, build upon this work non-commercially, and license their derivative works on different terms, provided the original work is properly cited, appropriate credit is given, any changes made indicated, and the use is non-commercial. See: http://creativecommons.org/licenses/by-nc/4.0/.

\section{REFERENCES}

1. European Commission. Dealing with the Impact of an Ageing Population in the EU (2009 Ageing Report): European Commission Brussels, 2009.

2. Mackun PJ, Wilson S, Fischetti TR, et al. Population distribution and change: 2000 to 2010: US Department of Commerce, Economics and Statistics Administration, US Census Bureau, 2011.

3. Colby SL, Ortman JM. Projections of the size and composition of the US population: 2014 to 2060: Population estimates and projections. Washington (DC): US Census Bureau, 2015.

4. Mathers CD, Stevens GA, Boerma T, et al. Causes of international increases in older age life expectancy. Lancet 2015;385:540-8.

5. Cho J, Martin P, Poon LW, et al. Successful aging and subjective well-being among oldest-old adults. Gerontologist 2015;55:132-43.

6. Levy B. Stereotype Embodiment: A Psychosocial Approach to Aging. Curr Dir Psychol Sci 2009;18:332-6.

7. Masoro EJ. Are age-associated diseases an integral part of aging? In: Masoro EJ, Austad SN, eds. Handbook of the Biology of Aging. Sixth Edition. New York: Academic Press, 2005:43-62.

8. Langer EJ. Counter clockwise: mindful health and the power of possibility. New York, NY, USA: Ballantine Books, 2009.

9. Levy BR. Mind matters: cognitive and physical effects of aging selfstereotypes. J Gerontol B Psychol Sci Soc Sci 2003;58:P203-11.

10. Nelson TD. Ageism: Stereotyping and prejudice against older persons: MIT press, 2004.

11. Rothermund K. Effects of age stereotypes on self-views and adaptation. In: Greve W, Rothermund K, eds. The adaptive self: personal continuity and intentional self-development. Cambridge: Hogrefe, 2005:223-42.

12. Levy BR, Slade MD, Murphy TE, et al. Association between positive age stereotypes and recovery from disability in older persons. JAMA 2012;308:1972-3.

13. Levy B, Banaji MR. Implicit ageism. Nelson TD, ed. Ageism: stereotyping and prejudice against older persons. Cambridge: MIT Press, 2002:49-75.

14. Levy BR, Zonderman AB, Slade MD, et al. Age stereotypes held earlier in life predict cardiovascular events in later life. Psychol Sci 2009;20:296-8.
15. Wurm S, Warner LM, Ziegelmann JP, et al. How do negative selfperceptions of aging become a self-fulfilling prophecy? Psychol Aging 2013;28:1088-97.

16. Levy B, Langer E. Aging free from negative stereotypes: successful memory in China and among the American deaf. J Pers Soc Psychol 1994;66:989-97.

17. Cook S, Marsiske M. Subjective memory beliefs and cognitive performance in normal and mildly impaired older adults. Aging Ment Health 2006;10:413-23.

18. Ercoli L, Siddarth P, Huang SC, et al. Perceived loss of memory ability and cerebral metabolic decline in persons with the apolipoprotein E-IV genetic risk for Alzheimer disease. Arch Gen Psychiatry 2006;63:442-8.

19. Levy BR, Ferrucci L, Zonderman AB, et al. A culture-brain link: Negative age stereotypes predict Alzheimer's disease biomarkers. Psychol Aging 2016;31:82-8.

20. Levy BR, Slade MD, Pietrzak RH, et al. Positive age beliefs protect against dementia even among elders with high-risk gene. PLoS One 2018;13:e0191004.

21. Sargent-Cox K, Anstey KJ. The relationship between agestereotypes and health locus of control across adult age-groups. Psychol Health 2015;30:652-70.

22. Langer EJ, Rodin J. The effects of choice and enhanced personal responsibility for the aged: a field experiment in an institutional setting. J Pers Soc Psychol 1976;34:191-8.

23. Rodin J, Langer EJ. Long-term effects of a control-relevant intervention with the institutionalized aged. $J$ Pers Soc Psychol 1977;35:897-902.

24. Abramson LY, Seligman ME, Teasdale JD. Learned helplessness in humans: critique and reformulation. J Abnorm Psychol 1978;87:49-74.

25. Langer EJ, Benevento A. Self-induced dependence. J Pers Soc Psychol 1978;36:886-93.

26. Langer E. Mindfulness: Addison-Wesley/Addison Wesley Longman, 1989.

27. Alexander CN, Langer E. Higher stages of human development: perspectives on adult growth: Oxford University Press, 1990.

28. Phillips D, Pagnini F. Health and the psychology of possibility. Critical mindfulness: Springer, 2016:173-82.

29. Folstein MF, Folstein SE, Fanjiang G. Mini-mental state examination: clinical guide: psychological assessment resources, 2002.

30. Creavin ST, Wisniewski S, Noel-Storr AH, et al. Mini-Mental State Examination (MMSE) for the detection of dementia in clinically unevaluated people aged 65 and over in community and primary care populations. Cochrane Database Syst Rev 2016:CD011145.

31. Guralnik JM, Simonsick EM, Ferrucci L, et al. A short physical performance battery assessing lower extremity function: association with self-reported disability and prediction of mortality and nursing home admission. J Gerontol 1994;49:M85-M94.

32. Miller EA, Weissert WG. Predicting elderly people's risk for nursing home placement, hospitalization, functional impairment, and mortality: a synthesis. Med Care Res Rev 2000;57:259-97.

33. Smits C, Kapteyn TS, Houtgast T. Development and validation of an automatic speech-in-noise screening test by telephone. Int J Audiol 2004;43:15-28.

34. Horner MD, Teichner G, Kortte KB, et al. Construct validity of the Babcock Story Recall Test. Appl Neuropsychol 2002;9:114-6.

35. Novelli G, Papagno C, Capitani E, et al. Tre test clinici di ricerca e produzione lessicale. Taratura su sogetti normali [Three clinical tests for lexical research and production. Calibration on regular subjects: Archivio di psicologia, neurologia e psichiatria, 1986.

36. Freides D, Avery ME. Narrative and visual spatial recall: assessment incorporating learning and delayed retention. Clin Neuropsychol 1991:5:338-44.

37. Della Sala S, Foley JA, Beschin N, et al. Assessing dual-task performance using a paper-and-pencil test: normative data. Arch Clin Neuropsychol 2010;25:410-9.

38. Revicki DA, Leidy NK, Howland L. Evaluating the psychometric characteristics of the Psychological General Well-Being Index with a new response scale. Qual Life Res 1996;5:419-25.

39. Ruini C, Ottolini F, Rafanelli C, et al. La validazione italiana delle Psychological Well-being Scales (PWB). Rivista di psichiatria 2003;38:117-30.

40. Pachana NA, Byrne GJ, Siddle $\mathrm{H}$, et al. Development and validation of the Geriatric Anxiety Inventory. Int Psychogeriatr 2007;19:103-14.

41. Yesavage JA, Brink TL, Rose TL, et al. Development and validation of a geriatric depression screening scale: a preliminary report. $J$ Psychiatr Res 1982;17:37-49.

42. Jongenelis K, Pot AM, Eisses AM, et al. Diagnostic accuracy of the original 30 -item and shortened versions of the Geriatric 
Depression Scale in nursing home patients. Int $\mathrm{J}$ Geriatr Psychiatry 2005;20:1067-74.

43. Levy BR, Slade MD, Kunkel SR, et al. Longevity increased by positive self-perceptions of aging. J Pers Soc Psychol 2002;83:261-70.

44. Laird NM. Missing data in longitudinal studies. Stat Med 1988;7:305-15.

45. McCulloch CE, Neuhaus JM. Generalized linear mixed models: Wiley Online Library, 2001.

46. Hofäcker D. In line or at odds with active ageing policies? Exploring patterns of retirement preferences in Europe. Ageing Soc 2015;35:1529-56.
47. World Health Organization. World report on ageing and health: World Health Organization, 2015.

48. Foster L, Walker A. Active and successful aging: a European policy perspective. Gerontologist 2015;55:83-90.

49. Merriam SB, Kee Y. Promoting community wellbeing: the case for lifelong learning for older adults. Adult Educ Q 2014;64:128-44.

50. Pagnini F, Philips D. Being mindful about mindfulness. Lancet Psychiatry 2015;2:288-9.

51. Engel GL. The need for a new medical model: a challenge for biomedicine. Science 1977;196:129-36. 